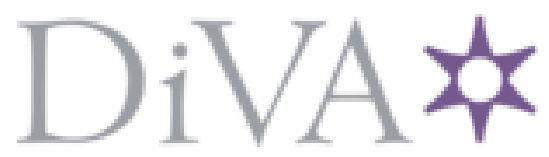

http://www.diva-portal.org

\title{
Preprint
}

This is the submitted version of a paper presented at IEEE Conference on Innovative Smart Grid Technologies (ISGT2014 North America).

Citation for the original published paper:

Armendariz, M., Chenine, M., Al-Hammouri, A., Nordström, L. (2014)

A Co-Simulation Platform for Medium/Low Voltage Monitoring and Control applications. In:

N.B. When citing this work, cite the original published paper.

Permanent link to this version:

http://urn.kb.se/resolve?urn=urn:nbn:se:kth:diva-13862 1 


\section{A Co-Simulation Platform for Medium/Low Voltage Monitoring and Control applications}

\author{
Mikel Armendariz, Moustafa Chenine, Lars \\ Nordström \\ Department of Industrial Information and Control Systems \\ School of Electrical Engineering, KTH - Royal Institute of \\ Technology \\ 100-44 Stockholm, Sweden \\ E-mail: mikela, moustafac, larsn \{@ics.kth.se\}
}

\author{
Ahmad Al-Hammouri \\ Department of Network Engineering and Security \\ Jordan University of Science and Technology \\ 22110 Irbid, Jordan \\ E-mail: Hammouri@just.edu.jo
}

\begin{abstract}
The increasing demand for electricity and the penetration of renewable energy resources into the electrical grid are triggering the electric power system to undergo changes and complex modifications at high, medium and low voltage levels. There are large and complex projects like Smart Grid Gotland that are developing solutions to deal with these challenges. However, there are several scenarios that could be more practical to be first studied at the simulation level. Therefore, the purpose of this study is to propose and develop a co-simulation platform that allows performing real-time monitoring and control tests and simulations for MV/LV grids. This platform combines both electrical and information and communication technology (ICT) infrastructure simulations. It consists of a real-time power system simulator (Opal-RT) connected to a communication network emulator (OPNET) through an automatic meter reading interface that is based on commercial off-the-shelf (COTS) and low cost devices like Raspberry $P i$ and Arduino boards. The platform allows the analysis of realistic MV/LV grids and communication networks and will facilitate the simulation and comparison of low voltage control strategies for real-time test scenarios that could be more complicated to test in the reality due to cost and complexity aspects (i.e. high power losses on the low voltage grid).
\end{abstract}

Index Terms - AMR, Co-simulation, Communication Network Emulator, DLMS/COSEM, MV/LV monitoring and control, Power System Simulator.

\section{INTRODUCTION}

The electric power system has evolved to a dynamic and complex system due to the increased demand and the utilization of renewable energy sources. This has forced the electric system to require reliable and accurate structures for monitoring and control. This requirement has caused an increased deployment of new types of measurement devices and supporting information and communication technology (ICT) infrastructures in order to better manage, monitor and control the electric power system. Due to cost considerations, considerable attention regarding monitoring and control has been focused at the top of this pyramidal structure, the high voltage/ medium voltage (HV/MV) substation. Thus, there is normally no remote control or measurement (SCADA systems) at the secondary substation and it is generally done off-line and using manual tap changers. However, the low voltage distribution grid (also called LV network) has the

This work is supported by the Swedish Energy Agency and Swedish Power Industry via SweGRIDS programme. potential to save and optimize the energy distribution. For instance, the roll-out of automatic meter readers (or remote meter reading devices) allows Distribution System Operators (DSO) to remotely monitor the consumer end of the LV grid for billing purposes. This roll-out opens new opportunities for innovative functions aiming at controlling and optimizing this part of the network.

The future power system will face challenges that have a serious impact on the LV grid in terms of monitoring and control [1], [2], For example: the introduction of electric vehicles, consumer installation of energy micro-production (Photovoltaic and Wind energy) as well as different market schemes for energy demand management (also known as Demand Side Management).

LV networks around the world lack observability and same situation occurs to consumers connected to them. This fact makes it challenging to study and assess the impacts of future scenarios in LV networks [3]. DSOs are aware of that lack of observability but adding additional devices to monitor the grid would suppose an extra cost. This is the reason why DSOs are showing interest in using the already deployed Automatic meter reading (AMR) infrastructure is this area [4], [5], [6].

\section{A. Scope of the paper}

The main objective of this paper is to introduce a cosimulation platform that allows the analysis of monitoring and control system applications for MV/LV electrical grids. Such a platform can facilitate the development and analysis of optimal information and communication systems based on realistic $\mathrm{MV} / \mathrm{LV}$ simulation scenarios with distributed energy resources (DER) and high power losses.

The platform utilizes commercial off-the-shelf (COTS) and low cost devices to replicate commercial metering infrastructure and DER communication and control solutions using conventional industry standards like DLMS/COSEM [7] and IEC 61850 [8].

It is intended to apply this platform to study realistic communication networks for MV/LV electrical grids that can affect the control part and to study and suggest control strategies to manage the $\mathrm{LV}$ voltage profile and the $\mathrm{MV} / \mathrm{LV}$ grid from the DSO perspective, in opposition to the Microgrid perspective [9].

\section{B. Outline}

The rest of the paper is organized as follows: In section II. an overview of challenges in $\mathrm{MV} / \mathrm{LV}$ grids are presented and $\mathrm{LV}$ grid monitoring is suggested along with potential $\mathrm{MV} / \mathrm{LV}$ 
applications. In Section III. the test platform for co-simulation of MV/LV monitoring and control applications is presented and its main components are explained. Section IV. shows the platform running a monitoring scenario and conclusions and future work are presented in Section V.

\section{Medium/Low Voltage Monitoring And Control SYSTEMS}

The electrical distribution network has traditionally been designed hierarchically, with the HV/MV substation at the top and dispersing the electricity towards a bulk of consumers through the LV network. The low voltage level of electricity distribution networks stretches from the secondary substation, via connection points, to the consumer meter and premises. The voltage levels at this substation are typically $10 / 20 \mathrm{kV}$ on the primary side and $1 / 0,4 \mathrm{kV}$ on the secondary side, depending on the size of the area served and the practices of the local utility (see Fig. 1).

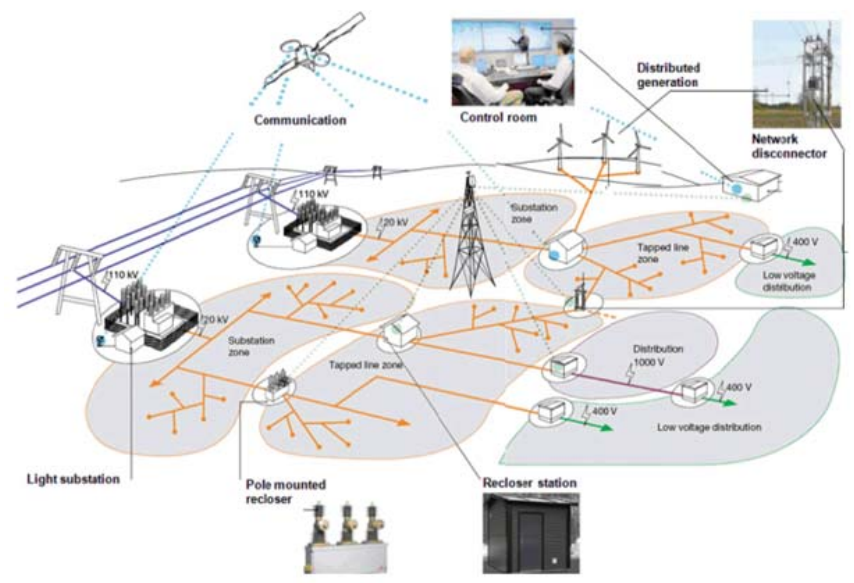

Fig. 1. MV grid from Smart Grid Gotland in Sweden.

\section{A. Challenges in $M V / L V$ grids}

The traditional electrical grid is undergoing changes and modifications in a similar way that cell phones have become "smart phones". In most cases, the power is obtained from a power plant (e.g. using fossil fuels, solar power, hydro-electric dam, etc.), then sent to transmission networks, then to distribution networks and finally delivered to the customers. The challenge arises when this traditional unidirectional procedure of power flow experiences structural changes and moves towards what it is called "Active Distribution Networks" [10], which adopts bidirectional energy flow between distribution networks and active consumers (prosumers), see Fig. 1. This novel concept encompasses the following characteristics:

- Large scale introduction of electric vehicles (EVs)

- Micro-production (PVs and wind turbines), distributed generation, microgrids

- Different market schemes for demand side management, etc.

For the traditional electrical grid the mentioned modifications and new assumptions can be considered as challenges that imply serious threats and influence in causing the following power quality problems:

- Voltage fluctuations due to PVs

- Unbalanced lines and grid instability
- Harmonics caused by some loads (e.g. motors, furnaces, etc.)

- Frequency variation

- Non-optimal voltage setting at the LV substation

- Outages, blackouts, etc.

As mentioned previously, due to the fact that the electrical grid has historically been performing in a "traditional" unidirectional way, it has not been required to thoroughly observe and control the LV levels of the distribution networks. However, this new power distribution concept requires further attention on LV monitoring and ICT systems to cope with the presented challenges.

\section{B. Benefits of LV grid monitoring}

The implementation of Automatic Meter Reading devices for grid monitoring and control can provide benefits for both utilities and customers. As detailed in [5], [6], [11] and [12] utilities will benefit by achieving a reduction in meter reads and associated management and administrative support, reduced equipment and equipment maintenance costs, reduced support expenses, faster restoration and shorter outages, better power quality related Key Performance Indicators (KPI) like System Average Interruption Duration Index (SAIDI) and System Average Interruption Frequency Index (SAIFI), increased meter reading accuracy, improved utility asset management, easier energy theft detection, and easier outage management. Customers on the other hand, will benefit by having billing accuracy improvements, lower number and shorter duration of outages, faster service restoration, flexible billing cycles and a variety of time-based rate options, and creating customer energy profiles for targeting Energy Efficiency/Demand Response programs.

\section{Potential MV/LV applications}

The integration of ICT systems into the electric power system fosters new application opportunities particularly at MV/LV levels. Some of these applications would require realtime communication (e.g. Power loss minimization through LV grid voltage profile control, zero fault detection, etc.), other applications would not require the communication to be so critical (e.g. network planning and maintenance support, power quality problem identification, consumed/generated energy reporting, etc.) and there are other applications that could be event based (e.g. proactive power outage identification and fault trace to locate operational/nonoperational customer clusters, remote electricity delivery switch on/off).

\section{THE CO-SIMULATION PLATFORM FOR MV/LV MONITORING AND CONTROL APPLICATIONS}

The performance and reliability of a MV/LV system can be verified in several ways. One method is to deploy the developed application in real scenarios and test the accuracy and performance of it through trial and error. This method is not always practical since power system as a vital and costly infrastructure is supposed to be available and in-service. Therefore, it is problematic to test critical scenarios like voltage fluctuations, brownouts and blackouts, communication failures, etc. A more desirable alternative is to establish a test platform which simulates the components and processes 
within the MV/LV system. This method allows applications to be tested before the costly deployment. In order to reflect this cross research discipline system consisting of several interconnected and interdependent components in cyber environment, each part of the whole system could be virtualized or modeled in corresponding simulator. The architecture of proposed platform is presented in this section.

\section{A. General Platform Architecture}

As shown in Fig. 2, two separate simulators can be used to model the power process and the communication network as the core of the MV/LV co-simulation platform. Power system simulator is expected to mimic the continuous time variant dynamics of the grid, while the communication network modeling is a discrete event-based simulation. The main challenging issue in terms of integrating simulators is the need to synchronize the simulators progress or events, specifically if the simulators are of different types, such as continuous time, in the case of the power system simulator and discrete event simulator in the case of the communication systems simulator. This problem can be avoided by using the real time simulators for both communication network and power system modeling and integrating them using another simulator or realtime interface, to exchange the relevant data, in this case the AMR metering infrastructure interface (Fig. 3).

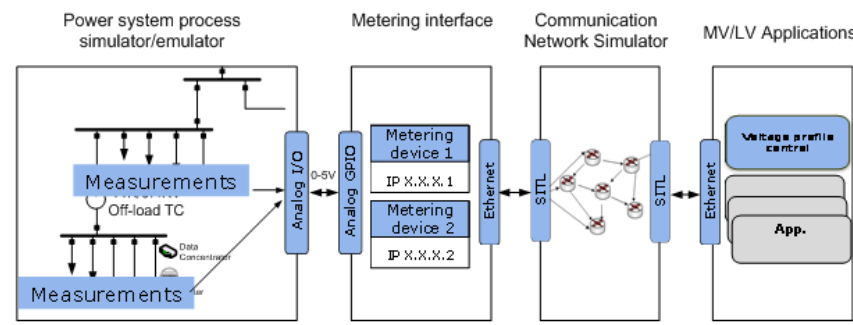

Fig. 2. Co-simulation platform for MV/LV applications

\section{B. Components of the Platform}

As shown in Fig. 2, the platform consists of four main components, i.e. a power system real time simulator, a group of low cost metering devices, a communication network simulator and a MV/LV control application. The measurement devices can be either hosted on the power system simulator or on an external platform linked to it. In order to test the general platform architecture, specific simulation environment or facilities are selected for each component of the platform.

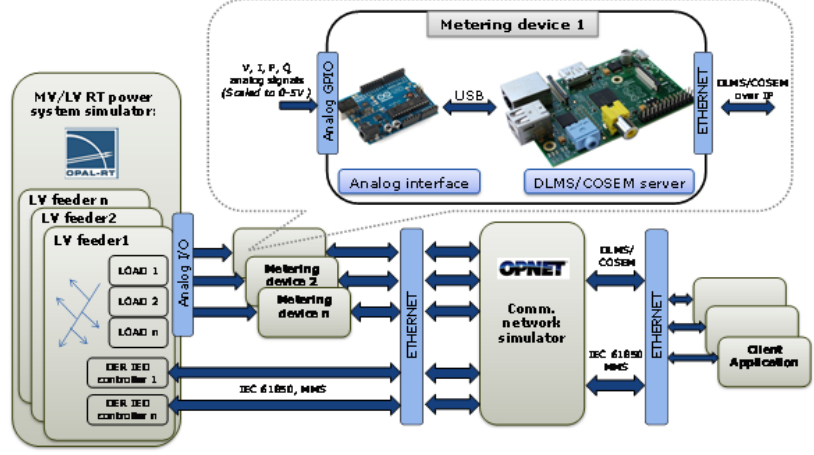

Fig. 3. Components in the co-simulation platform

1) Power System Simulator: Simulink and OPAL-RT

The power system process modeling can be implemented by several types of simulators and the main requirement to be considered is the ability of the simulation to be real time. For this platform, OPAL-RT eMEGAsim simulator [13] is selected to simulate the power system in real-time. This simulator is built around a Spartan3 FPGA Reconfigurable I/O Node. One of the advantages of using OPAL-RT is that it the models can be built using Matlab/Simulink, this also allows running the models offline (i.e. not in real time) for testing purposes.

If these MV/LV grid models are based on real electricity networks, the simulated scenarios (e.g. voltage instabilities, frequency variations, voltage quality problems due to harmonics, power losses, outages, islanding mode operation, etc.) will be more realistic and it will help to create the basis for understanding the typical problems in the current MV/LV grid and its required level of monitoring and control. Therefore, the simulated grid topology is chosen to be based on the grid employed by the Smart Grid Gotland project [14] (see Fig. 1), which is a $R \& D$ project that intends to demonstrate how cost effective and new smart grid technologies can improve the electrical quality of large rural grids by utilizing large quantities of distributed and renewable energy sources (RES) and advanced load management strategies. Fig. 4 shows an example of a MV/LV grid model used to perform simulations to test the platform. This grid is based on the $10 \mathrm{kV}$ rural distribution network in Smart Grid Gotland project.

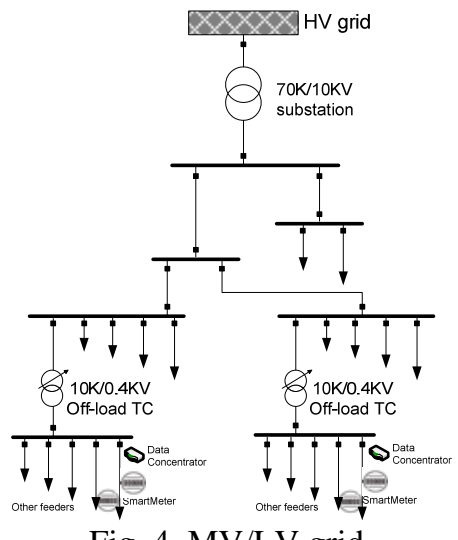

Fig. 4. MV/LV grid

\section{2) Interface: $A M R$ infrastructure}

Automatic meter reading (AMR) or smart metering refers to the act of recording energy-related data and by using telemetry systems transmitting that data to the utility, which processes the information for monitoring and billing purposes [15]. That data can be electricity, gas or hot/cold water related data. In the case of electricity, typical measurements are current (A), voltage (V), power consumption (kW) and active energy consumption (kWh), which can be read on hourly basis [16]. [17] shows the data exchange for meter reading, tariff and load control specified by International Electrotechnical Commission (IEC). There are several standards for meter data exchange (e.g. IEC 61107, Euridis, MBUS, ANSI C12.18, etc.). [18] shows the state-of the-art for smart metering communication standards and concludes that the current leading standard for meter data exchange is DLMS/COSEM (or IEC 62056); its main benefit is the interoperability aspect [19]. IEC together with DLMS User Association [20] works on the smart meter standardization to help utilities to move 
from proprietary protocols to standard protocols. DLMS stands for Device Language Message Specification and it is an application layer specification, independent of the lower layers and thus of the communication channel. It is designed to support messaging to and from metering devices. Whereas COSEM stands for COmpanion Specification for Energy Metering, it is an interface model of communicating metering devices and it provides a view of the functionality available through the communication interfaces. It has an objectoriented approach and the following specifications are required to understand the protocol [21]:

IEC 62056-61 defines the Object identification system (OBIS codes) that provides standard identification codes to identify each quantity in the metering domain (e.g. Current [A] - 1.1.31.7.0.255, Voltage [V] - 1.1.32.7.0.255, Power [kW] - 1.1.21.7.0.255 and Positive active energy $(\mathrm{A}+)$ in phase L1 total [kWh] - 1.1.21.8.0.255, etc.).

IEC 62056-62 defines the interface classes (IC) for the objects that share similar attributes and methods. There is a large number of ICs, for example IC 1 data class for instantaneous quantities like the billing period counter, IC 3 register class for quantities like energy that require the value and the unit, IC 4 extended register classes that require also the timestamp, IC 7 profile generic classes to model historic values like voltage profiles, etc. Each meter can have several instantiated classes (e.g. different profile generics for I, V, P, A+ in L1, etc.) but with a different and unique OBIS code.

IEC-62056-53 defines the COSEM Application layer.

IEC 62056-46 defines the data link layer using HDLC protocol and IEC 62056-47 defines the COSEM transport layers for IPv4 networks. These DLMS/COSEM specifications are fully described in the DLMS UA coloured books [22], [23], [24], [25].

DLMS/COSEM protocol follows a client/server paradigm, acting the meter as the server and the data collection system as the client.

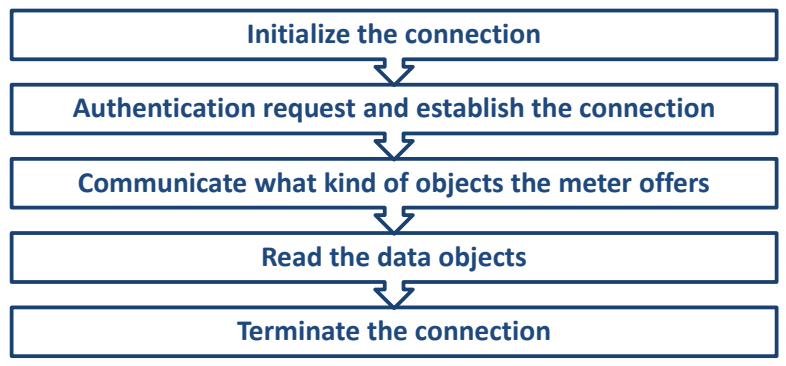

Fig. 5. DLMS/COSEM based Server-Client connection

Fig. 5 shows the communication sequence for DLMS/COSEM based Server-Client communication. For further details consult www.dlms.com/faq.

The AMR infrastructure implemented for this platform is based on the metering device shown in Fig. 6. This device is built around a Raspberry Pi computer [26]. The Raspberry Pi is a low cost single-board device, which is run by a modified version of Debian Linux (called Raspbian) optimized for the ARM architecture. Its GPIO are purely digital, therefore an Arduino Uno [27] board is added as slave I/O board to be able to perform analogue sampling of $\mathrm{V}, \mathrm{I}, \mathrm{P}$ and $\mathrm{Q}$ signals provided by the power system simulator. The ArduinoRaspberry Pi communication is carried out via USB

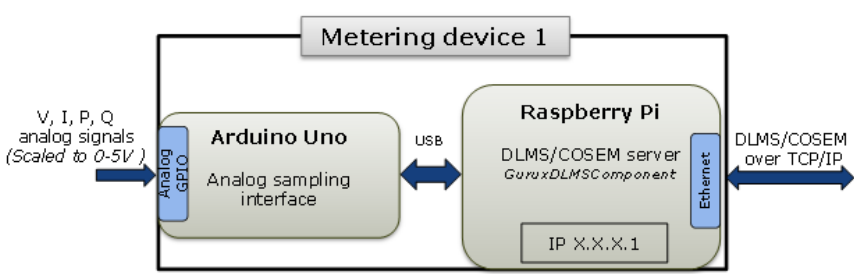

Fig. 6. The main modules of the AMR Infrastructure

Each Raspberry Pi hosts the Gurux DLMS component for Java [28], which is an open source DLMS/COSEM compliant server that enables to create a DLMS meter. The client application is also open source and can be run in a Windows or Linux based machine. For this example a 64bit Win7 PC was used to run an client application with a user interface.

\section{3) Communication Systems Simulator: OPNET}

Automated metering infrastructure as envisioned for supporting LV monitoring and control will be reliant on the underlying communication infrastructure. In order to analyze the performance and behavior of the communication networks, several kinds of simulation software are available such us OPNET, OMNET or ns-2. In this study, some important factors are considered to choose the communication simulation environment; first, the ability of adding hardware in the loop of simulation, second, an accurate and powerful tool which reflects the available networking components in real world as detail as possible while still keeps its simplicity in terms of modeling, extendibility and finally ability of simulation in real time.

OPNET Modeler provides a comprehensive development environment in order to model communication networks and distributed systems. Both behavior and performance of modeled systems can be analyzed by performing discrete event simulations. The OPNET Modeler environment includes tools for all phases of a study, including model design, simulation, data collection, and data analysis. In comparison to other network simulation tool such as ns-2, the features on OPNET fulfill the aforementioned requirements of the cosimulation platform for MV/LV applications, such as real time simulation, extendibility or simplicity in modeling (visual support) in a more convenient way. The detailed comparisons of available network simulator's performance in different scenarios are presented in [29], [30].

The OPNET System-in-the-Loop module (SITL) makes it possible to connect the simulation model to live network hardware by providing interfaces or gateways. SITL module provided the ability of exchanging packets with the external hardware via an Ethernet link while the simulation is running in real-time.

Each SITL module inside the simulation environment is assigned to a specific network adaptor on the machine to insert the packets received on that network interfaces into the simulation. Assigned network adaptor can be a real interface or virtual one. In this developed platform, certain number of virtual network interface is created to be connected into SITL gateways.

\section{PROOF OF CONCEPT USING THE PLATFORM}

At present time, with the purpose of testing the platform and showing that it works all the way from the power system 
simulator to the $\mathrm{MV} / \mathrm{LV}$ monitoring application part, GuruxDLMS Director [31] open source client application is executed to monitor the voltage values of a selected load.

Fig. 7 shows few snapshots of the components that form the platform: the power system simulator, the metering equipment, the communication network simulator and a client application.

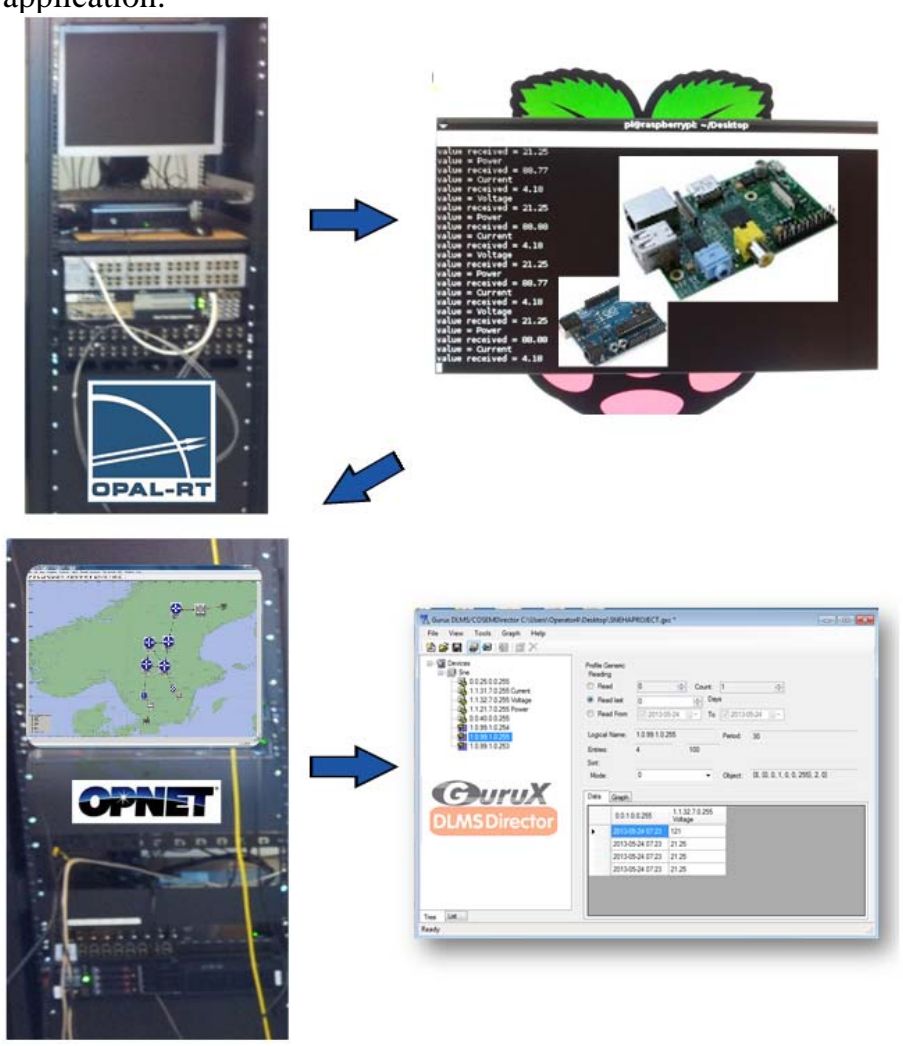

Fig. 7. A snapshot of the MV/LV monitoring platform

\section{CONCLUSION AND FUTURE WORK}

A real-time co-simulation test-bed has been proposed for MV/LV networks that employs COTS, low cost devices and open source code for $\mathrm{LV}$ monitoring. This platform is planned to be used to simulate $\mathrm{MV} / \mathrm{LV}$ grids and communication networks, create scenarios with high power losses and propose and compare control strategies to manage the voltage profile and reduce the losses by inserting control devices like tap changers (OLTC) in secondary substations and to control ancillary services like end-user PV inverters, energy storage, EVs, etc. applying IEC 61850 standard.

It is intended in the future to connect the platform to SCADA-DMS systems and explore possible interoperability, cyber security and reliability related issues.

\section{REFERENCES}

[1] J. Fan, S. Borlase, "The evolution of distribution”, Power and Energy Magazine, IEEE Vol. 7, Issue 2, pp. 63-68, March-April 2009

[2] M. Bayegan, "A Vision of the Future Grid”, Power Engineering Review, IEEE Vol. 21, Issue 12, pp. 10-12, Dec. 2001

[3] Luis F. Ochoa, Pierluigi Mancarella, "Low-Carbon LV Networks: Challenges for Planning and Operation”, Power and Energy Society General Meeting, 2012 IEEE, San Diego, CA

[4] S. Willing et al., "Improving quality of supply and usage of assest in distribution grids by introducing a Smart Operator", CIRED2013, paper no. 0718, Stockholm, June 2013
[5] L. Garpetun, P. Nylen; "Benefits from Smart Meter Investments"; CIRED 22nd International Conference on Electricity Distribution; Stockholm June 2013

[6] L. Garpetun;”Improved network analysis by using data from smart meters"; CIRED 22nd International Conference on Electricity Distribution; Stockholm June 2013

[7] “DLMS user association”, 2013. [Online]. Available: www.DLMS.com

[8] "Power Utility Automation - IEC 61850", International Electrotechnical Commission, Edition 6.2, 2002. Geneva, Switzerland: IEC

[9] N. Hatziargyriou et al., "Microgrids - Large Scale Integration of Microgeneration to Low Voltage Grids”, paper C6-309, CIGRE 06, Paris, August 2006

[10] S. Chowdhury, S.P. Chowdhury and P. Crossley. Microgrids and Active Distribution Networks. London, UK. The Institution of Engineering and Technology 2009. Print.

[11] L. Garpetun; "Improved network analysis by using hourly meter values"; Integration of Renewables into the Distribution Grid, CIRED 2012; pp. 1-4.

[12] "Advanced Metering Infrastructure (AMI)", Electric Power Research Institute (EPRI), February 2007, Palo Alto, CA

[13] “eMEGAsim”, OpalRT Technologies, 2011. [Online]. Available: http://www.opalrt.com/sites/default/files/OPB1_emegasimbrochure_DA14_hires.pdf

[14] GEAB, Vattenfall, ABB, KTH, "Smart Grid Gotland Pre-Study", Smart Grid Gotland 2011. [Online]. Available: http://www.smartgridgotland.se/pdf/sgg_forstudie.pdf

[15] Wikipedia contributors, "Automatic Meter Reading," Wikipedia, http://en.wikipedia.org/wiki/Automatic_meter_reading (accessed August 19, 2013).

[16] L. Garpetun, "Smart meters make for a smarter grid", Vattenfall R\&D Magazine, No.1, February 2013

[17] "Electricity metering - Data exchange for meter reading, tariff and load control. Part 61: Object Identification System (OBIS) “, IEC 6205661:2002. [Online]. Available: www.iec.ch.

[18] K. De Craemer, G. Deconinck, "Analysis of State-of-the-art Smart Metering Communication Standards", Proceedings of the 5th Young Researchers Symposium, Leuven, Belgium, March 2010

[19] W. Strabbing,"Smart meter interoperability and interchangeability in Europe”, European Smart Metering Industry Group (ESMIG). 2013. [Online]. article/?searchterm=None

[20] “DLMS user association”, 2013. [Online]. Available: www.DLMS.com

[21] Vinoo S Warrier, G. Prasanth ,"Implementing DLMS Client and Server Protocols in Meters, IED's, MRI's and Meter Reading Applications An Overview”, 2009. [Online]. Available: http://gopalakrishnanprasanth.wordpress.com/article/implementingdlms-client-and-server-3bk0x32fl4sfh-15/

[22] Companion specification for Energy Metering, "Identification system and interface classes,” in Blue Book, DLMS User Association, 19972010.

[23] Companion Specification for Energy Metering, "DLMS/COSEM architecture and protocols,” in Green Book, DLMS User Association, 1997-2009

[24] Companion Specification for Energy Metering, "DLMS/COSEM architecture and protocols," in Yellow Book, DLMS User Association, 1997-2010

[25] Companion Specification for Energy Metering, "DLMS/COSEM architecture and protocols," in White Book, DLMS User Association, 2003

[26] "Raspberry Pi," Raspberry Pi Foundation, 2013. [Online]. Available: http://www.raspberrypi.org

[27] "Arduino UNO," Arduino, 2013. [Online]. Available: http://arduino.cc/en/Main/arduinoBoardUno

[28] “ComponenGuruxDLMS4Java_2.0.5”, Gurux Ltd, 2013. [Online]. Available: http://www.gurux.fi/index.php?q=Downloads

[29] P. Trúchly P., et al., "Simulation of IMS using current simulators", IEEE International Symposium ELMAR, 2008

[30] Lee, T., Shon, S., "Development of real-time simulator theory and modeling technique based on NS-2", International Conference on Education Technology and Computer, 2010.

[31] "GuruxDLMS Director”, Gurux Ltd, 2013. [Online]. Available: http://www.gurux.fi/index.php?q=Downloads 